\title{
Modeling of Orientation-Dependent Photoelastic Constants in Cubic Crystal System
}

\author{
Lubna Jahan Rashid Pinky', Shakila Islam', Md. Nur Kutubul Alam¹, \\ Mohammad Arif Hossain'2, Md. Rafiqul Islam1 \\ ${ }^{1}$ Department of Electrical and Electronic Engineering, Khulna University of Engineering \& Technology, Khulna, \\ Bangladesh \\ ${ }^{2}$ Department of Mathematics, Khulna University of Engineering \& Technology, Khulna, Bangladesh \\ Email: islambit@yahoo.com
}

Received 3 January 2014; revised 5 February 2014; accepted 19 February 2014

Copyright (C) 2014 by authors and Scientific Research Publishing Inc.

This work is licensed under the Creative Commons Attribution International License (CC BY). http://creativecommons.org/licenses/by/4.0/

(c) (i) Open Access

\section{Abstract}

Euler's rotation theorem and tensor rotation technique are applied to develop a generalized mathematical model for determining photoelastic constants in arbitrary orientation of cubic crystal system. Two times rotations are utilized in the model relating to crystallographic coordinates with Cartesian coordinates. The symmetry of photoelastic constants is found to have strong dependence with rotation angle. Using the model, one can determine photoelastic constants in any orientation by selecting appropriate rotation angle. The outcome of this study helps to characterize spatial variation of residual strain in crystalline as well as polycrystalline materials having cubic structure using the experimental technique known as scanning infrared polariscope.

\section{Keywords}

Mathematical Modeling; Photoelastic Constants; Arbitrary Crystal Orientation; Euler's Rotation Theorem; Tensor Rotation Technique

\section{Introduction}

The quality of semiconductor materials as well as devices is strongly dependent on residual strain induced in the materials during the growth and cooling processes [1]-[3]. Yamada et al. [4] [5] have developed computer controlled scanning infrared polariscope (SIRP) that is used to measure spatial distribution of strain in crystalline semiconductor wafers and ingot. Although SIRP can be effectively utilized for residual strain mapping in crys- 
talline materials [6] [7], it can not be applied to measure residual strain in polycrystalline materials, particularly, in solar cell materials [8]. The main problem is the photoelastic constants which are the key parameters required for SIRP measurements [6] [7] and have crystal direction/orientation dependence [9]. The photoelastic constants $P_{11}$ and $P_{12}$, and $P_{44}$ are measured by applying stress parallel to $<100>$, and $<111>$ directions [10] [11]. To the best of our knowledge, there is no experimental measurement of photoelastic constants in arbitrary crystal direction.

It is well known that some mechanical and optical properties of semiconductor materials and devices strongly depend on crystal orientation [12] [13]. The polycrystalline Si-based solar cells exhibit poor efficiency that is speculated to be due to residual strain induced in the grain boundary as reported so far [8]. However, the quantitative amount of strain can not be measured due to lacking of orientation-dependent photoelastic constants of Si. Although Fukuzawa et al. [14] have reported spatial distribution of strain in polycrystalline Si using SIRP, determination of photoelastic constants in arbitrary crystal direction was not reported in details.

In this study, we for the first time propose a generalized mathematical model to determine photoelastic constant in arbitrary crystal orientation combining Euler's rotation theorem and tensor rotation technique. Using the model one can calculate different components of photoelastic constants in cubic crystals just by choosing appropriate rotation angle. Herein the model is applied to determine the orientation-dependent photoelastic constants in Si crystal as an example. However, the model developed in the present study can be used to determine photoelastic constants in cubic/Zincblende crystal structure.

\section{Proposed Model}

The model is developed with the combination of Euler's rotation theorem [15] and tensor rotation technique [9]. The rotation schematic is shown in Figure 1 where two times rotations are performed for the versatility of the model. At first $X$ axis is rotated in $X Y$ plane by an angle $\varphi$ about the $Z$ axis so that $Y$ axis is rotated in $-X Y$ plane by the same angle. After the first rotation, the new position of $X, Y$, and $Z$ are denoted by $X^{\prime}, Y^{\prime}$, and $Z^{\prime}$. Again, the $X^{\prime}$ axis is rotated in $X^{\prime} Z^{\prime}$ plane about the $Y^{\prime}$ axis by an angle $\theta$, as a result, the $Z^{\prime}$ axis is also rotated in $-X^{\prime} Z^{\prime}$ plane by the same angle. The final position of $X^{\prime \prime}$ and $Z^{\prime \prime}$ is obtained after the second rotation. If Cartesian coordinate system relates to the conventional crystal coordinate system, the rotation angles $\varphi$ and $\theta$ of the general [hkl] direction relative to a crystal coordinate system fixed onto the [100], [010], and [001] directions can be given by the following relationships [16]

$$
\begin{aligned}
& \tan \varphi=\frac{k}{h} \\
& \tan \theta=\frac{\sqrt{h^{2}+k^{2}}}{l}
\end{aligned}
$$

where the indices $h, k$, and $l$ are the real integers for the case when the crystalline direction is specified in terms of the angles $\varphi$ and $\theta$. We can determine the photoelastic constants in any crystal direction/orientation with the combination of the angles $\varphi$ and $\theta$.

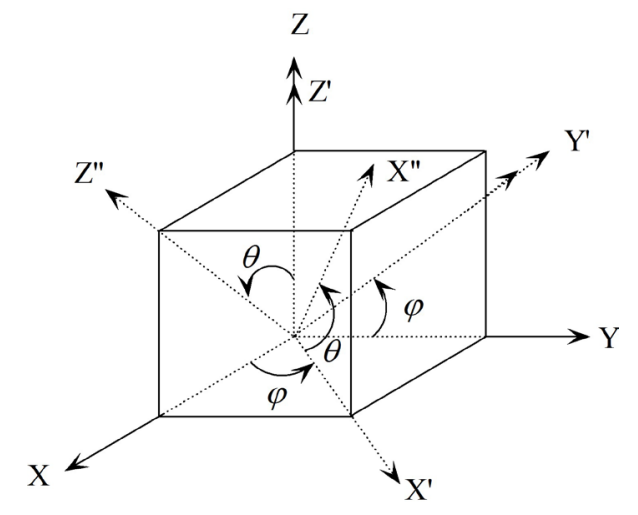

Figure 1. Rotation schematics applied in the proposed model. 


\section{Mathematical Analysis}

At first, transformation of $X Y$ plane about $Z$ axis by an angle $\varphi$ and then transformation of $X^{\prime} Z^{\prime}$ plane about $Y^{\prime}$ axis by an angle $\theta$ is applied according to the schematics shown in Figure 1. The rotation matrices obtained from the first and second rotations are represented by $C$ and $B$, respectively, and indicated by Equations (3) and (4)

$$
\begin{array}{cccc} 
& X & Y & Z \\
X^{\prime} & \cos \varphi & \sin \varphi & 0 \\
C=Y^{\prime} & -\sin \varphi & \cos \varphi & 0 \\
Z^{\prime} & 0 & 0 & 1 \\
& X^{\prime} & Y^{\prime} & Z^{\prime} \\
X^{\prime \prime} & \cos \theta & 0 & \sin \theta \\
B=Y^{\prime \prime} & 0 & 1 & 0 \\
Z^{\prime \prime} & -\sin \theta & 0 & \sin \theta
\end{array}
$$

According to Euler's rotation theorem, the combined rotation matrix M can be given [15] by

$$
M=B C=\left[\begin{array}{ccc}
\cos \theta \cos \varphi & \cos \theta \sin \varphi & \sin \theta \\
-\sin \varphi & \cos \varphi & 0 \\
-\sin \theta \cos \varphi & -\sin \theta \sin \varphi & \cos \theta
\end{array}\right]
$$

The photoelastic constant, $P_{i j k l}$, stress, $X_{i j}$, and change in refractive index, $\Delta n$ in semiconductor materials can be expressed by [9]

$$
\Delta n=-P_{i j k l} X_{i j}
$$

Expansion of Equation (6) yields $81 P_{i j k l}$ components. Details are available in [9]. According to tensor rotation/transformation rule, symmetry transformation results in equivalent 36 components form the 81 components [9]-[11]. Employing an additional symmetry for the cubic system, only three independent components of photoelastic constants $P_{11}, P_{12}$, and $P_{44}$ are existed [9]. The independent components along with other components are summarized in Table 1. If photoelastic constant in any direction is known, its value in unknown direction can be determined using the following tensor rotation rule [9],

$$
P_{\text {mnop }}^{\prime}=\sum_{i, j, k, l=1} a_{m n} a_{n j} a_{o k} a_{p l} P_{i j k l}
$$

where $a_{m i}, a_{n j}, a_{o k}$, and $a_{p l}$ are the respective direction cosines between the two sets of coordinate axes before and after symmetry transformation in the case of Cartesian tensors which are expressed in the form of rotation matrix shown in Equation (5). $P_{i j k l}$ is the value of known photoelastic constant and $P_{m n o p}^{\prime}$ indicates the value which is to be evaluated. By expanding Equation (7) and considering the symmetry described in Table 1 [9], the independent components of photoelastic constants in rotated direction can be expressed by

Table 1. Equivalent components of $P_{i j k l}$ for cubic crystal system [9].

\begin{tabular}{lccccc}
\hline$P_{11}$ & $P_{12}$ & $P_{12}$ & 0 & 0 & 0 \\
\hline$P_{12}$ & $P_{11}$ & $P_{12}$ & 0 & 0 & 0 \\
$P_{12}$ & $P_{12}$ & $P_{11}$ & 0 & 0 & 0 \\
0 & 0 & 0 & $P_{44}$ & 0 & 0 \\
0 & 0 & 0 & 0 & $P_{44}$ & 0 \\
0 & 0 & 0 & 0 & 0 & $P_{44}$ \\
\hline
\end{tabular}




$$
\begin{aligned}
P_{1111}^{\prime}= & P_{11}\left[\cos ^{4} \theta\left(\cos ^{4} \varphi+\sin ^{4} \varphi\right)+\sin ^{4} \theta\right]+2 P_{12}\left[\cos ^{4} \theta \cos ^{2} \varphi \sin ^{2} \varphi+\cos ^{2} \theta \sin ^{2} \theta\right] \\
& +P_{44}\left[\cos ^{4} \theta \cos ^{2} \varphi \sin ^{2} \varphi+\cos ^{2} \theta \sin ^{2} \theta\right] \\
P_{2222}^{\prime}= & P_{11}\left[\cos ^{4} \varphi+\sin ^{4} \varphi\right]+2 P_{12}\left[\cos ^{2} \varphi \sin ^{2} \varphi\right]+P_{44}\left[\cos ^{2} \varphi \sin ^{2} \varphi\right] \\
P_{3333}^{\prime}= & P_{11}\left[\sin ^{4} \theta\left(\cos ^{4} \varphi+\sin ^{4} \varphi\right)+\cos ^{4} \theta\right]+2 P_{12}\left[\sin ^{4} \theta \cos ^{2} \varphi \sin ^{2} \varphi+\cos ^{2} \theta \sin ^{2} \theta\right] \\
& +P_{44}\left[\sin ^{4} \theta \cos ^{2} \varphi \sin ^{2} \varphi+\cos ^{2} \theta \sin ^{2} \theta\right] \\
P_{1122}^{\prime}= & P_{2211}^{\prime}=2 P_{11}\left[\cos ^{2} \varphi \sin ^{2} \varphi\right]+P_{12}\left[\cos ^{2} \theta\left(\cos ^{4} \varphi+\sin ^{4} \varphi\right)+\sin ^{2} \varphi\right]-P_{44}\left[\cos ^{2} \varphi \sin ^{2} \varphi \cos ^{2} \theta\right] \\
P_{1133}^{\prime}= & P_{3311}^{\prime}=P_{11}\left[\sin ^{2} \theta \cos ^{2} \theta\left(\cos ^{4} \varphi+\sin ^{4} \varphi+1\right)\right]+P_{12}\left[2 \cos ^{2} \theta \sin ^{2} \theta \cos ^{2} \varphi \sin ^{2} \varphi+\cos ^{4} \theta+\sin ^{4} \theta\right] \\
& -P_{44}\left[\cos ^{2} \theta \sin ^{2} \theta-\cos ^{2} \varphi \sin ^{2} \varphi \cos ^{2} \theta \sin ^{2} \theta\right] \\
P_{2233}^{\prime}= & P_{3322}^{\prime}=2 P_{11}\left[\sin ^{2} \theta \cos ^{2} \varphi \sin ^{2} \varphi\right]+P_{12}\left[\sin ^{2} \theta\left(\cos ^{4} \varphi+\sin ^{4} \varphi\right)+\cos ^{2} \theta\right]-P_{44}\left[\cos ^{2} \varphi \sin ^{2} \varphi \sin ^{2} \theta\right] \\
P_{1212}^{\prime}= & 2 P_{11}\left[\cos ^{2} \theta \cos ^{2} \varphi \sin ^{2} \varphi\right]-2 P_{12}\left[\cos ^{2} \theta \cos ^{2} \varphi \sin ^{2} \varphi\right]+P_{44}\left[\sin ^{2} \theta \sin ^{2} \varphi+\cos ^{4} \varphi \cos ^{2} \theta\right] \\
P_{2323}^{\prime}= & 2 P_{11}\left[\cos ^{2} \theta \cos ^{2} \varphi \sin ^{2} \varphi\right]-2 P_{12}\left[\sin ^{2} \theta \cos ^{2} \varphi \sin ^{2} \varphi\right]+P_{44}\left[\sin ^{2} \theta \sin ^{4} \varphi+\cos ^{4} \varphi \cos ^{2} \theta\right] \\
P_{3131}^{\prime}= & P_{11}\left[\sin ^{2} \theta \cos ^{2} \theta\left(\cos ^{4} \varphi+\sin ^{4} \varphi+1\right)\right]+2 P_{12}\left[\cos ^{2} \theta \sin ^{2} \theta \cos ^{2} \varphi \sin ^{2} \varphi-\cos ^{2} \theta \sin ^{2} \theta\right] \\
& +P_{44}\left[\cos ^{4} \theta \cos ^{2} \varphi+\cos ^{2} \varphi \sin ^{2} \varphi \cos ^{2} \theta \sin ^{2} \theta+\sin ^{4} \theta \sin ^{2} \varphi\right]
\end{aligned}
$$

\section{Orientation Dependent Photoelastic Constant}

Using the mathematical formulations derived in Equations (8) to (16), we have calculated orientation-dependent photoelastic constants from [100] to [010], [110] to [001], and [100] to [001] directions with the combination of rotation angles $\varphi$ and $\theta$. The results are determined for the semiconductor Si using the known values of photoelastic constants listed in Table 2.

Figures 2(a)-(c) show a comparison among the photoelastic constants $P_{1111}^{\prime}, P_{2222}^{\prime}$, and $P_{3333}^{\prime}$ which are determined for the rotation angles $\varphi=0^{\circ}$ to $90^{\circ}$ and $\theta=0^{\circ}, \varphi=0^{\circ}$ and $\theta=0^{\circ}$ to $90^{\circ}$, and $\varphi=45^{\circ}$ and $\theta=0^{\circ}$ to $90^{\circ}$, respectively. To compare their magnitudes, the figures are plotted in the same scale. The components $P_{1111}^{\prime}$ and $P_{2222}^{\prime}$ are found symmetrical in Figure 2(a), but in Figure 2(b), $P_{1111}^{\prime}$ and $P_{3333}^{\prime}$ are symmetrical and their values are maximum at $45^{\circ}$ that is in the [110] direction. Apart from $45^{\circ}$ they decrease following Gaussian profile and become equal to the known component $P_{11}$ in the [100], [010], and [001] directions. It is also found in Figures 2(a) and (b) that the components $P_{3333}^{\prime}$ and $P_{2222}^{\prime}$ are independent of the rotation angles and their values are equal to $P_{11}$. The variations of the same components of photoelastic constants are shown in Figure 2(c) for the rotation from [110] to [001] direction. It is found that the components $P_{1111}^{\prime}$ and $P_{2222}^{\prime}$ are symmetrical at [110] direction. In contrast, $P_{2222}^{\prime}$ and $P_{3333}^{\prime}$ are symmetrical at [001] direction. Apart from these directions the cubic symmetry of these components has been lost due to the rotation. It is also found in Figure 2(c) that the component $P_{2222}^{\prime}$ is independent of the rotation angle. On the other hand, the components $P_{1111}^{\prime}$ and $P_{3333}^{\prime}$ vary nonlinearly and their magnitudes change oppositely from -0.0593 to -0.1053 due to the rotation.

Table 2. Experimental measurement of Photoelastic constants $P_{11}, P_{12}$, and $P_{44}$ for Si crystal taken from Ref.
[10].
\begin{tabular}{|cccc}
\hline Photoelastic constants & $P_{11} X||<100>$ & $P_{12} X||<100>$ & $P_{44} X||<111>$ \\
\hline & -0.1053 & 0.0137 & -0.054 \\
\hline
\end{tabular}



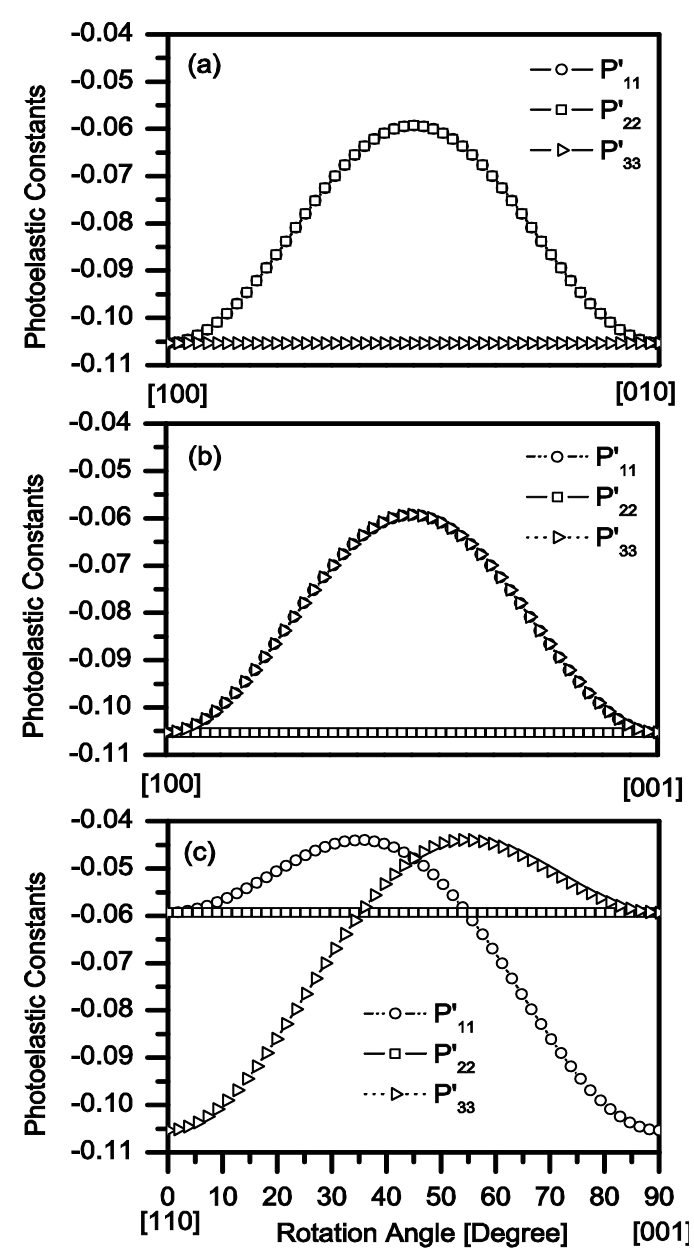

Figure 2. Variation of orientation-dependent photoelastic constants $P_{1111}^{\prime}, P_{2222}^{\prime}$, and $P_{3333}^{\prime}$ plotted for the directions from (a) [100] to [010]; (b) [110] to [001]; (c) [100] to [001].

The variations of the photoelastic constants $P_{1122}^{\prime}, P_{2211}^{\prime}, P_{1133}^{\prime}, P_{3311}^{\prime}, P_{2233}^{\prime}$, and $P_{3322}^{\prime}$ are shown in Figures 3(a)-(c) for the same rotation angles and directions as mentioned above. The curves are also presented in the same scale for making a comparison among them. It is found in Figure 3(a) that the components $P_{1133}^{\prime}$, $P_{3311}^{\prime}, P_{2233}^{\prime}$, and $P_{3322}^{\prime}$ are symmetrical and their values are equal to the component $P_{12}$. Similar results are found for the components $P_{1122}^{\prime}, P_{2211}^{\prime}, P_{2233}^{\prime}$, and $P_{3322}^{\prime}$ in Figure $3(\mathrm{~b})$. In contrast, the components $P_{1122}^{\prime}$, $P_{2211}^{\prime}, \quad P_{1133}^{\prime}$, and $P_{3311}^{\prime}$ vary symmetrically following Gaussian distribution similar to that shown in Figures 3(a) and (b). The maximum value is found -0.0323 for the components $P_{1122}^{\prime}$ and $P_{2211}^{\prime}$, and $P_{1133}^{\prime}$ and $P_{3311}^{\prime}$ in the directions [110] and [101], respectively. The direction dependent photoelastic constants $P_{1122}^{\prime}, P_{2211}^{\prime}, P_{1133}^{\prime}$, $P_{3311}^{\prime}, \quad P_{2233}^{\prime}$, and $P_{3322}^{\prime}$ is shown in Figure 3(c) where cubic symmetry in these components has been lost due to rotation, but the symmetry between $P_{1122}^{\prime}$ and $P_{2211}^{\prime}, P_{1133}^{\prime}$ and $P_{3311}^{\prime}$, and $P_{2233}^{\prime}$ and $P_{3322}^{\prime}$ are found. The magnitudes of the components $P_{1122}^{\prime}$ and $P_{2211}^{\prime}$, and $P_{2233}^{\prime}$ and $P_{3322}^{\prime}$ vary from -0.0323 to 0.0137 , and 0.0137 to -0.0323 , respectively. As seen in Figure $3(\mathrm{c})$ that the profiles of the components $P_{1133}^{\prime}$ and $P_{3311}^{\prime}$ follow Gaussian distribution and their magnitude is equal to 0.0137 in the directions [110] and [001]. The magnitude of these components is also found to be -0.0162 in the direction [111].

Figures 4(a)-(c) show a comparison among the orientation-dependent photoelastic constants $P_{1212}^{\prime}, P_{2323}^{\prime}$, and $P_{3131}^{\prime}$ where their profiles are found identical for the rotation from [100] to [010] and [110] to [001] with respect to the profiles obtained from [110] to [001]. As seen in Figures 3(a) and (c), the cubic symmetry of these components has been lost due to the rotation from [100] to [010] and [110] to [001] directions. On the other 

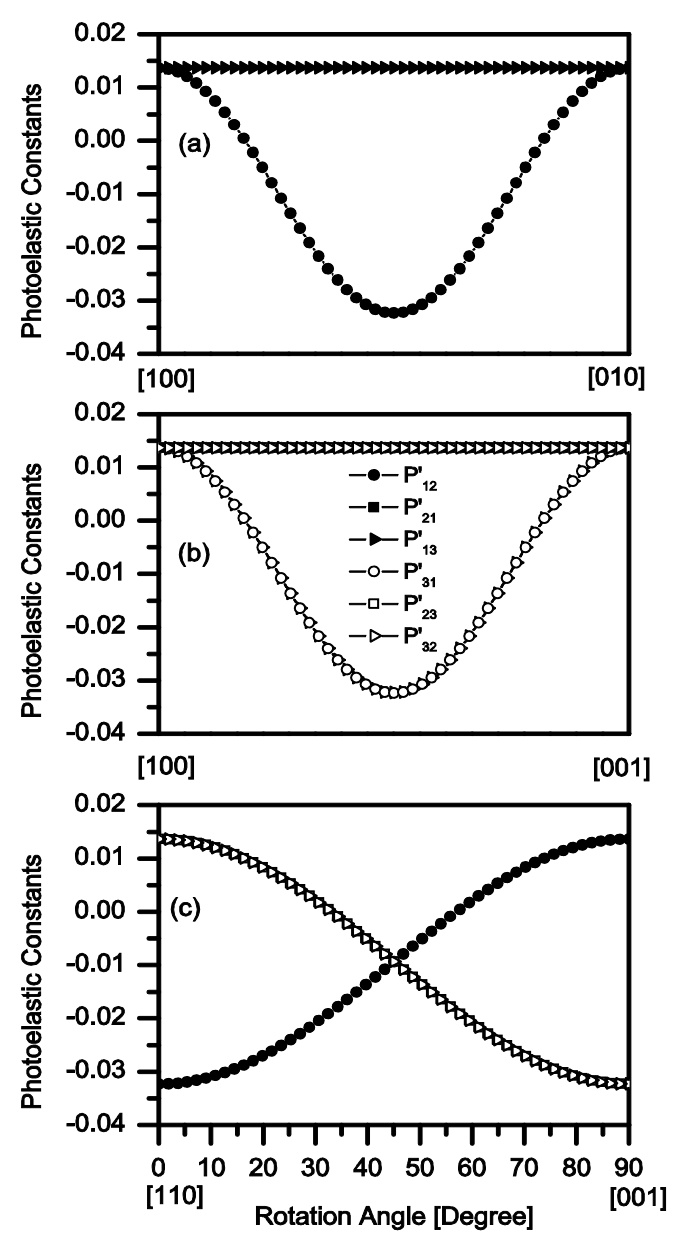

Figure 3. Variation of orientation-dependent photoelastic constants $P_{1122}^{\prime}, P_{2211}^{\prime}, P_{1133}^{\prime}, P_{3311}^{\prime}$, $P_{2233}^{\prime}$, and $P_{3322}^{\prime}$ plotted for the directions from (a) [100] to [010]; (b) [110] to [001]; (c) [100] to [001]. The magnitude of the photoelastic constants $P_{1122}^{\prime}$ $=P_{2211}^{\prime}, P_{1133}^{\prime}=P_{3311}^{\prime}$, and $P_{2233}^{\prime}=P_{3322}^{\prime}$.

hand, the component $P_{3131}^{\prime}$ is found asymmetrical with respect to the components $P_{1212}^{\prime}$ and $P_{2323}^{\prime}$ in Figure 3(b). The magnitudes of $P_{1212}^{\prime}, P_{2323}^{\prime}$, and $P_{3131}^{\prime}$ are equal to the known component $P_{44}$ at [100], but their magnitudes are evaluated $-0.073,-0.06615$, and -0.027 , respectively, at [110] direction. The components $P_{1212}^{\prime}$ and $P_{2323}^{\prime}$ are evaluated -0.027 and $P_{3131}^{\prime}$ is -0.073 at [101] direction. Further, their magnitudes are found to be very small at [010] and [001] directions. In Figure 3(c), the components $P_{1212}^{\prime}, P_{2323}^{\prime}$, and $P_{3131}^{\prime}$ are estimated $-0.073,-0.06615$, and -0.027 , respectively, at [110] direction. On the other hand, their values are evaluated $-0.0428,-0.0361$, and -0.0582 , respectively, at [111] direction. Furthermore, the values of $P_{1212}^{\prime}$, and $P_{3131}^{\prime}$ are estimated -0.027 , and $P_{2323}^{\prime}$ is -0.0203 at [001] direction.

\section{Conclusion}

A generalized mathematical model is developed for cubic crystal system to determine photoelastic constants in arbitrary orientation with the combination of tensor rotation technique and Euler's rotation theorem. Three independent components of photoelastic constants become nine independent components due to two times rotations. However, some of them are found symmetrical depending on the rotation direction. The magnitude and variation pattern of the photoelastic constants are also found to have direction-dependent. But, for a particular 

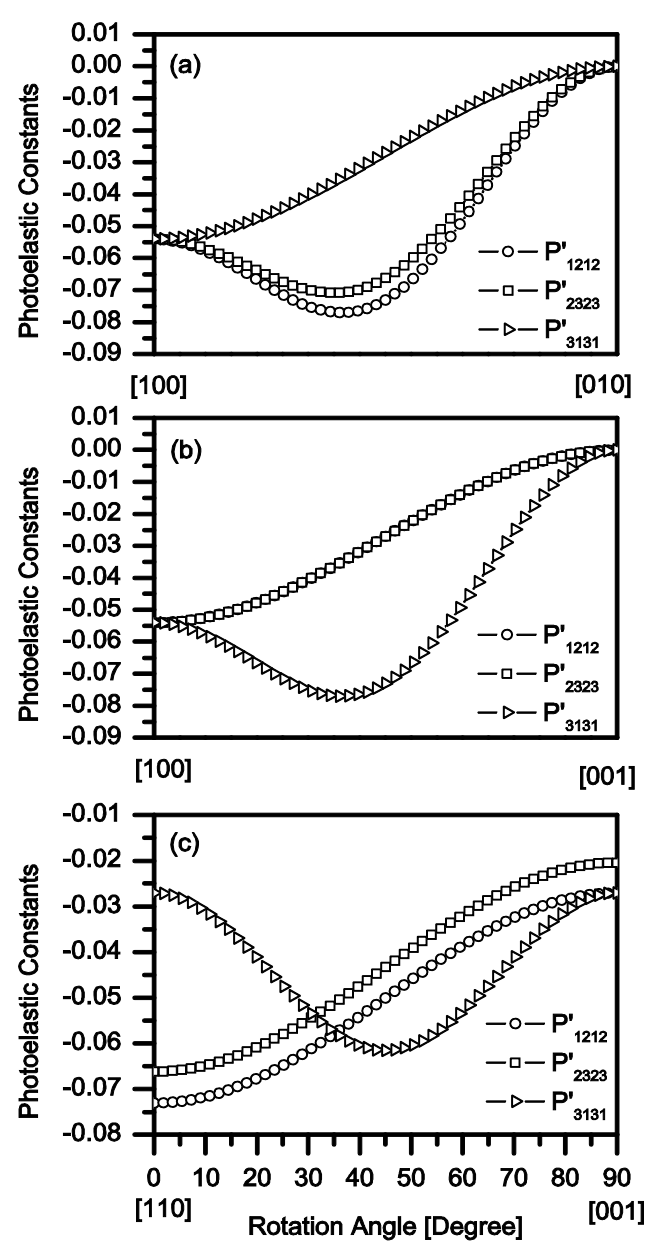

Figure 4. Variation of orientation-dependent photoelastic constants $P_{1212}^{\prime}, P_{1313}^{\prime}$, and $P_{2323}^{\prime}$ plotted for the directions from (a) [100] to [010], (b) [110] to [001], and (c) [100] to [001].

direction, some components are found independent of rotation angle. Here, the model is applied for silicon crystal as an example. It can be applied for any crystal having cubic/Zincblende structure.

\section{References}

[1] Yamada, M. (1993) Relief of Residual Strains in Gallium Phosphide (100) Wafers by Cracking. Journal of Applied Physics, 74, 6435. http://dx.doi.org/10.1063/1.355125

[2] Islam, M.R., Verma, P., Yamada, M., Kodama, S., Hanaue, Y. and Kinoshita K. (2002) The Influence of Residual Strain on Raman Scattering in $\mathrm{In}_{\mathrm{x}} \mathrm{Ga}_{1-\mathrm{x}}$ As Single Crystals. Materials Science and Engineering: B, 91-92, 66-69. http://dx.doi.org/10.1016/S0921-5107(01)00972-2

[3] Miao, Z.L., Yu, T.J., Xu, F.J., Song, J., Lu, L., Huang, C.C., Yang, Z.J., Wang, X.Q., Zhang, G.Y., Zhang, X.P., Yu, D.P. and Shen, B. (1994) Dependence of Optical Gain on Crystal Orientation in Surface-Emitting Lasers with Strained Quantum Wells. Applied Physics Letters, 65, 1886. http://dx.doi.org/10.1063/1.112878

[4] Yamada, M. (1993) High-Sensitivity Computer-Controlled Infrared Polariscope. Review of Scientific Instruments, 64, 1815. http://dx.doi.org/10.1063/1.1144016

[5] Chu, T., Yamada, M., Donecker, J., Rossberg, M., Alex, V. and Riemannb H. (2003) Optical Anisotropy in Dislocation-Free Silicon Single Crystals. Microelectronic Engineering, 66, 327-332. http://dx.doi.org/10.1016/S0167-9317(02)00935-8

[6] Hermsm, M., Fukuzawa, M., Melov, V.G., Schreiber, J., Mock, P. and Yamada, M. (2000) Residual Strain in Annealed 
GaAs Single-Crystal Wafers as Determined by Scanning Infrared Polariscopy, X-Ray Diffraction and Topography. Journal of Crystal Growth, 210, 172-176. http://dx.doi.org/10.1016/S0022-0248(99)00673-9

[7] Fukuzawa, M. and Yamada, M. (1996) Fine Structures of Residual Strain Distribution in Fe-Doped lnP-100) Wafers Grown by the LEC and VCZ Methods. Journal of Electronic Materials, 25, 337-342. http://dx.doi.org/10.1007/BF02666598

[8] Chen, J., Chen, B., Sekiguchi, T., Fukuzawa, M. and Yamada, M. (2008) Correlation between Residual Strain and Electrically Active Grain Boundaries in Multicrystalline Silicon. Applied Physics Letters, 93, 112105. http://dx.doi.org/10.1063/1.2983649

[9] Narasimhamutry, T.S. (1981) Photoelastic and Electro-Optic Properties of Crystals. Plenum Press, New York. http://dx.doi.org/10.1007/978-1-4757-0025-1

[10] Biegelsen, D.K. (1974) Physical Review Letters, 32, 1196.

[11] Levine, Z.H., Zhong, H., Wei, S., Allan, D.C. and Wilkins, J.W. (1992) Strained Silicon: A Dielectric-Response Calculation. Physical Review B, 45, 4131. http://dx.doi.org/10.1103/PhysRevB.45.4131

[12] Hasan, M.M., Islam, M.R. and Teramoto, K. (2012) Crystallographic Orientation-Dependent Optical Properties of GaInSb Mid-Infrared Quantum Well Laser. Optik—International Journal for Light and Electron Optics, 123, 19931997. http://dx.doi.org/10.1016/j.ijleo.2011.09.021

[13] Zwinge, G., Wehmann, H.-H., Schlachetzki, A. and Hsu C.C. (1993) Orientation-Dependent Growth of InGaAs/InP for Applications in Laser-Diode Arrays. Journal of Applied Physics, 74, 5516. http://dx.doi.org/10.1063/1.354208

[14] Fukuzawa, M., Yamada, M., Islam, M.R., Chen, J. and Sekiguchi, T. (2012) Quantitative Photoelastic Characterization of Residual Strains in Grains of Multicrystalline Silicon. Journal of Electronic Materials, 39, 700-703. http://dx.doi.org/10.1007/s11664-010-1164-x

[15] http://mathword.wolfram.com/Euler Angles.html

[16] Okuno, Y., Uomi, K., Aoki, M. and Tsuchiya, T. (1997) Direct Wafer Bonding of III-V Compound Semiconductors for Free-Material and Free-Orientation Integration. IEEE Journal of Quantum Electronics, 33, 959-969. http://dx.doi.org/10.1109/3.585484 